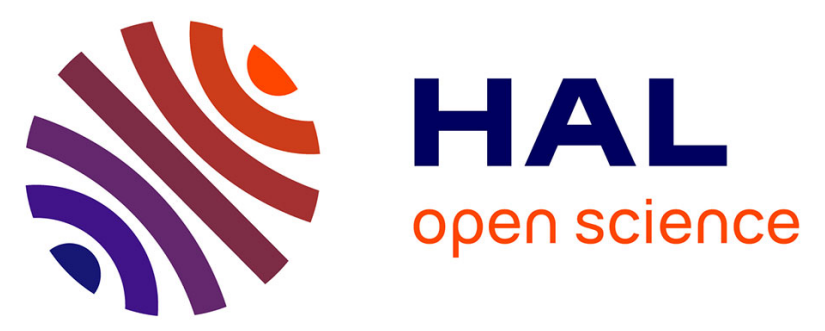

\title{
Photosynthèse et élaboration des réserves des graines chez divers types de sojas à croissance indéterminée, semi-déterminée et déterminée
}

\author{
Jean Calmés, Noël Gelfi, Guy Viala, Gérard Cavalié, Robert Blanchet
}

\section{- To cite this version:}

Jean Calmés, Noël Gelfi, Guy Viala, Gérard Cavalié, Robert Blanchet. Photosynthèse et élaboration des réserves des graines chez divers types de sojas à croissance indéterminée, semi-déterminée et déterminée. Agronomie, 1987, 7 (1), pp.33-40. hal-00884967

\section{HAL Id: hal-00884967 \\ https://hal.science/hal-00884967}

Submitted on 1 Jan 1987

HAL is a multi-disciplinary open access archive for the deposit and dissemination of scientific research documents, whether they are published or not. The documents may come from teaching and research institutions in France or abroad, or from public or private research centers.
L'archive ouverte pluridisciplinaire HAL, est destinée au dépôt et à la diffusion de documents scientifiques de niveau recherche, publiés ou non, émanant des établissements d'enseignement et de recherche français ou étrangers, des laboratoires publics ou privés. 


\title{
Photosynthèse et élaboration des réserves des graines chez divers types de sojas à croissance indéterminée, semi-déterminée et déterminée
}

\author{
Jean CALMÉS, Noël GELFI $\left({ }^{*}\right)$, Guy VIALA, Gérard CAVALIÉ \& Robert BLANCHET $\left({ }^{*}\right)$ \\ Centre de Physiologie végétale, Unité Associée au C.N.R.S. $n^{\circ}$ 24l, Université Paul Sabatier, 118, route de Nar- \\ bonne. 31062 Toulouse Cedex \\ (*) I.N.R.A. Station d'Agronomie, Centre de Recherches de Toulouse, B.P. 12, 31320 Castanet-Tolosan.
}

\begin{abstract}
Une étude comparative du développement, de la photosynthèse et de l'élaboration des réserves des graines de soja (Glycine $\max (\mathrm{L}$.$) , Merr.) est réalisée pour deux variétés à croissance indéterminée : " Kingsoy " la plus cul-$ tivée en France et « $\mathrm{L} 65$ » lignée non nodulante, une lignée à croissance semi-déterminée « 59-9-1-2 》 et une variété de type déterminé, dit demi-nain, « Hobbit ». Le type de croissance n’influe pas sur la photosynthèse du couvert végétal ni sur la production des graines; la lignée non nodulante est déficiente en $\mathrm{N}$ : son activité assimilatrice est plus faible et sa productivité réduite.

Les assimilats synthétisés durant la période de remplissage ne suffisent pas à assurer le développement des graines qui nécessite une redistribution des constituants biochimiques de l'appareil végétatif. Ainsi la protéogenèse dans les graines bénéficie largement de la mobilisation de molécules carbonées et azotées préalablement stockées. En revanche, la formation des lipides dépend davantage du carbone fixé pendant la période de remplissage. L'alimentation déficiente en azote de la lignée « L 65 » se traduit par une synthèse lipidique plus importante et une moindre teneur en protéines. Le rapport protéines/lipides des graines apparaît beaucoup plus conditionné par l'alimentation azotée que par le type de croissance.
\end{abstract}

Mots clés additionnels : Transferts, lipides, protéines, production.

Additional key words : Translocation, lipids, proteins, production.

\section{INTRODUCTION}

Le carbone fixe par photosynthèse au cours du cycle végétatif est la source des chaînons carbonés nécessaires à l'élaboration des réserves des graines. Les glucides, lipides et protéines qu'elles contiennent sont formés, soit à partir des photoassimilats de la feuille, soit par suite de la redistribution des composés stockés dans les organes végétatifs. La participation respective de ces deux sources de carbone aux synthèses lipidiques et protéiques des graines est encore mal connue. Chez le soja, de nombreux travaux concernent le transfert des assimilats (PATE, 1980 ; LAYZELL \& LA RuE, 1982 ; Grima-PetTENATI, 1985 ; NAUdy- 
de SERRES, 1985 ; SERRES et al., 1985 ; EGLI et al., 1985). D'autres se rapportent à la protéogenèse dans les graines (CALMÉS et al., 1983 ; SALADO-NAVARRO et al., 1985) ou à leur métabolisme lipidique (MAZLIAK, 1968 ; RUBEL et al., 1972 ; OHLROGGE \& KUO, 1984) qui s'avère très intense entre les 15 et $45^{\mathrm{e}}$ jours suivant la floraison (PRIVETT et al., 1973).

Ce travail, réalisé sur des sojas cultivés au champ, appartenant à des variétés classiques en France, à croissance indéterminée, ou à des cultivars nouveaux de type semi-déterminé ou déterminé (VIDAL \& HANAFIAH, 1985), se propose d'examiner l'élaboration des réserves des graines chez ces trois types, différents par leurs morphologies et leurs modes de développement. Pendant la phase de remplissage, il précise, en particulier, le devenir du carbone fixé et sa répartition entre les fractions glucidique, lipidique et protéique de la graine. Il apporte également des informations sur les capacités de production des divers types étudiés.

\section{MÉTHODES EXPÉRIMENTALES}

\section{A. Matériel végétal et conditions de culture}

L'expérimentation est conduite durant la période du grossissement des graines. Quatre variétés de soja sont étudiées comparativement: deux sont à croissance indéterminée et de groupe de précocité II : " Kingsoy », le plus cultivé en France, et une lignée «L 65 » non nodulante provenant d'Urbana (U.S.A.), dont le phénotype est très voisin de « Kingsoy » lorsqu'elle est bien alimentée en $\mathrm{N}$ (BouNIOLS et al., 1985). La troisième est de type déterminé, dit «demi-nain » (COOPER, 1981), d'origine américaine et de groupe de précocité III, «Hobbit »; le quatrième est une lignée obtenue par VIDAL (I.N.R.A., Montpellier) à croissance semi-déterminée, « 59-9-1-2 », plus précoce que « Kingsoy ».

Les quatre variétés, semées le 19 avril 1985, sont cultivées au champ, en parcelles de $30 \mathrm{~m}^{2}$, avec une irrigation satisfaisant sensiblement l'évapotranspiration maximale. Le sol brun, limono-argileux profond, bien fertilisé en phosphore et potassium, est inoculé avec Rhizobium japonicum, souche G 49, incorporée au sol avant semis. Il renferme, en profondeur, environ $200 \mathrm{~kg}$. ha ${ }^{-1}$ d'azote minéral provenant de reliquats de cultures céréalières antérieures. Cependant la nodulation n'en a pas été affectée.

\section{B. Techniques utilisées}

Les mesures de photosynthèse nette du couvert sont réalisées selon la méthode de WELLS et al. (1982), en caisson transparent à couvercle amovible couvrant $0,5 \mathrm{~m}^{2}$, dans lequel l'air est constamment renouvelé pendant la mesure en maintenant une légère surpression dans le caisson. La quantité de $\mathrm{CO}_{2}$ fixée est déterminée d'après la différence des teneurs de l'air, entre l'entrée et la sortie du caisson, et le débit gazeux. La photosynthèse nette des feuilles du som- met du couvert est mesurée, à l'aide d'une petite chambre comparable à celle de SHIMSHI (1969). Ces mesures sont effectuées en fin de matinées ensoleillées sur deux points différents de chaque parcelle.

Conformément à l'approche de WeLls et al. (1982), une estimation de la quantité globale du $\mathrm{CO}_{2}$ fixé pendant la période de remplissage des graines est obtenue en multipliant la photosynthèse nette du couvert par la durée de l'insolation et en intégrant cette valeur depuis le début du remplissage jusqu'à la maturité. Cette quantité est ensuite exprimée en équivalentsglucose. La masse des graines est également convertie en équivalents-glucose en appliquant les coefficients indiqués par PENNING de VRIES et al. (1974), qui représentent les quantités de glucose nécessaires à la synthèse des principales catégories chimiques d'un organe : 1,2 $\mathrm{g}$ de glucose pour $1 \mathrm{~g}$ de glucides, $3 \mathrm{~g}$ pour $1 \mathrm{~g}$ de lipides, $2,7 \mathrm{~g}$ en moyenne pour $1 \mathrm{~g}$ de protéines dans le cas de sojas nodulés $(2,5 \mathrm{~g}$ pour «L 65 », lignée non nodulante qui utilise uniquement des nitrates). Comme cela a été discuté par ailleurs (BLANCHET et al., 1982), il s'agit d'une approximation intéressante au plan agronomique.

Les incorporations de ${ }^{14} \mathrm{CO}_{2}$ sont effectuées selon des techniques précédemment décrites (CALMÉS et al., 1985), la foliole centrale bien éclairée est placée durant $30 \mathrm{mn}$ dans une enceinte de $400 \mathrm{~cm}^{3}$ où l'on fait dégager $3,5 \mathrm{MBq}$ de ${ }^{14} \mathrm{CO}_{2}$. Après $30 \mathrm{mn}$ de charge, la radioactivité résiduelle de l'enceinte est très faible ; sa mesure permet de connaître la quantité de ${ }^{14} \mathrm{CO}_{2}$ fixé par la feuille. Le matériel végétal traité le matin vers $8 \mathrm{~h}$ (T.U.) est récolté, soit en fin de journée ( $8 \mathrm{~h}$ après l'incorporation), soit trois jours plus tard ( $72 \mathrm{~h}$ après l'incorporation). Il est conservé au froid à $-18^{\circ} \mathrm{C}$.

La répartition du radiocarbone entre les composés glucidiques, lipidiques et azotés est estimée dans les graines situées à l'aisselle de la feuille alimentée en ${ }^{14} \mathrm{CO}_{2}$.

Le protocole expérimental utilisé pour l'extraction des lipides est une variante des méthodes préconisées par FISHWICK \& WRIGHT (1977) : il est basé d'abord sur l'action du butanol, puis du mélange chloroformeméthanol. Trois graines, soit $1 \mathrm{~g}$ de matière fraîche environ, sont broyées au Polytron dans $20 \mathrm{ml}$ de $\mathrm{n}$ butanol. Le broyat est porté, durant $10 \mathrm{mn}$, dans un bain-marie bouillant. Après refroidissement et centrifugation, le surnageant est décanté : il contient les lipides solubles dans le butanol. Le culot est ensuite soumis à deux extractions successives au mélange chloroforme-méthanol $(2: 1, \mathrm{v} / \mathrm{v})$ : agitation durant $15 \mathrm{mn}$ dans $20 \mathrm{ml}$ de solvant, centrifugation et récupération du surnageant. Après réunion des trois surnageants et évaporation à sec, les lipides bruts ainsi obtenus sont repris avec $10 \mathrm{ml}$ de chloroformeméthanol et lavés deux fois avec $3 \mathrm{ml} \mathrm{d}$ 'une solution aqueuse contenant du $\mathrm{KCl}, 0,1 \mathrm{M}$ et de l'acide acétique (1 p. 100) (MURPHY \& LEECH, 1981).

Le culot est soumis ensuite à trois extractions aqueuses: les surnageants obtenus et les eaux de lavage des lipides bruts sont réunis et concentrés : ils renferment les composés hydrosolubles. Les protéines contenues dans le culot résiduel sont hydrolysées par $\mathrm{HCl} 6 \mathrm{~N}$ et les acides aminés libérés fixés sur résines Dowex 50. 


\section{RÉSULTATS}

\section{A. Croissance, développement et production}

Les données rassemblées dans les tableaux 1 et 2 permettent de comparer le déroulement du cycle de développement, les caractéristiques morphologiques et le rendement des quatre variétés retenues. On constate (tabl. 1) que la floraison débute à des dates assez voisines et se poursuit pendant un mois environ, chez les quatre génotypes. Cependant, l'allongement des gousses et le début du remplissage des graines, marqués par l'apparition des premières graines atteignant $3 \mathrm{~mm}$, interviennent plus tôt chez «59-9-1-2 》 et « Hobbit ». Dans tous les cas, ils correspondent sensiblement à la fin de la croissance de la tige principale. La phase de constitution des réserves est ainsi la plus longue chez «Hobbit» (durée d'ensoleillement : 810 h) ; elle est au contraire courte pour « 59-9-1-2 », qui bénéficie néanmoins du même ensoleillement que «L 65 » $(620 \mathrm{~h})$, celui de « Kingsoy » étant un peu supérieur $(650 \mathrm{~h})$.

L'examen du tableau 2 montre la double influence de la nodulation et du type de croissance sur le développement végétatif. Pour la lignée " L 65 », le diamètre et la hauteur de la tige, ainsi que le nombre de nœuds, sont réduits comparativement à " Kingsoy ».
Il a été constaté, par ailleurs, que, à la mi-floraison, les feuilles de « $\mathrm{L} 65$ » renferment, par rapport à la matière sèche, seulement 2,1 p. 100 d'azote au lieu de 5,1 chez les variétés nodulées. Ces dernières présentent les caractères de chacun des types : hauteur et nombre de nœuds décroissant de "Kingsoy » à « Hobbit ", quoique la tige principale demeure robuste. La croissance de cette tige s'achève vers la mi-floraison, chez " Hobbit » et « 59-9-1-2 », et se poursuit jusqu'au voisinage de la fin-floraison pour les types indéterminés qui sont caractérisés par la production simultanée d'organes végétatifs et reproducteurs.

Par ailleurs, des mesures d'indice foliaire ont été effectuées. Pour les variétés nodulées il croît d'une valeur de 4 à la floraison jusqu'à un maximum proche de 7 au début du mois d'août, ensuite il diminue lentement. Pour la variété non nodulante, il est nettement plus faible : 3 à la floraison, 5 pendant le développement des graines.

La production en graines et en matière sèche totale de la lignée non nodulante « L 65 » est faible. Parmi les variétés nodulées, "Kingsoy » élabore la plus grande quantité de biomasse, mais, son indice de récolte étant inférieur, la production de graines est très voisine (en masse et en nombre) de celle des deux autres génotypes. Les gousses des sojas de types déterminé et semi-déterminé sont moins nombreuses mais elles renferment plus de graines, aussi les rendements sont-ils sensiblement identiques.

TABLEAU I

Chronologie du développement de sojas à type de croissance indéterminée (lignée " $L 65$ " non nodulante et variété "Kingsoy ») semidéterminée (lignée « 59-9-1-2 ») et déterminée (variété « Hobbit »). Les durées d'insolation pendant la période de remplissage des graines sont respectivement $620,650,620$ et $810 \mathrm{~h}$; semis le 19 avril.

Chronology of soybean development for two indeterminate genotypes ( $L 65$ » non-nodulating and " Kingsoy »), one semideterminate (line (59-9-1-2》) and one determinate genotype ( $($ Hobbit 》) during seed filling. Insolation duration was respectively 620, 650, 620 and $810 \mathrm{~h}$. Sowing, April 19

\begin{tabular}{|c|c|c|c|c|}
\hline & L 65 & Kingsoy & $59-9-1-2$ & Hobbit \\
\hline Début floraison & $26-06$ & $06-07$ & $26-06$ & $02-07$ \\
\hline Premières graines $(3 \mathrm{~mm})$ & $03-08$ & $30-07$ & $10-07$ & $16-07$ \\
\hline Fin croissance, tige principale & $05-08$ & $30-07$ & $16-07$ & $17-07$ \\
\hline Fin floraison & $05-08$ & $07-08$ & $27-07$ & $30-07$ \\
\hline Maturité & $15-10$ & $15-10$ & $20-09$ & $20-10$ \\
\hline
\end{tabular}

\section{TABLEAU 2}

Principaux caractères agronomiques concernant le développement végétatif et la fructification. Moyenne de 4 lots de 12 plantes \pm écart type. La quantité totale d'azote absorbé, calculée d'après les teneurs de matière sèche, est de $21,2 \pm 1,8 \mathrm{~g} . \mathrm{m}^{-2}$ pour « $L$ 65 », 49,2 $\pm 2,1$ pour « Kingsoy 》, 39,2 \pm 3,2 pour « 59-9-1-2 » et 47,3 \pm 5,6 pour « Hobbit ».

Main agronomic characters of growth and fruiting. Mean of 4 replicates of 12 plants $\pm S D$. Total uptake of nitrogen, calculated from dry matter content, was $21.2 \pm 1.8 \mathrm{~g} . \mathrm{m}^{-2}$ for « $L 65 », 49.2 \pm 2.1$ for « Kingsoy », $39.2 \pm 3.2$ for « 59-9-1-2 » and $47.3 \pm 5.6$ for « Hobbit ».

\begin{tabular}{|c|c|c|c|c|c|c|}
\hline \multirow[b]{2}{*}{ Diamètre du collet $(\mathrm{mm})$} & \multicolumn{2}{|c|}{ L 65} & Kingsoy & $59-9-1-2$ & \multicolumn{2}{|c|}{ Hobbit } \\
\hline & 4,0 & 1,3 & $5,6 \pm$ & $7,8 \pm$ & 6,0 & 1,4 \\
\hline Hauteur finale $(\mathrm{cm})$ & 84 & 25 & $134 \pm 23$ & $88 \pm$ & 55 & \pm \\
\hline Nombre total de nœuds & 14,4 & 7,0 & $17,9 \pm 3,8$ & $14,0 \pm$ & 11,8 & 1,3 \\
\hline Nombre de nœuds fructifères & 7,6 & $\pm \quad 4,1$ & $10,3 \pm 3,8$ & $11,0 \pm 1,7$ & 6,7 & $\pm \quad 0,8$ \\
\hline Nombre de gousses. $\mathrm{m}^{-2}$ & 650 & \pm 220 & $1400 \pm 150$ & $1260 \pm 130$ & 1150 & \pm 110 \\
\hline Nombre de graines. $\mathrm{m}^{-2}$ & 1680 & \pm 130 & \pm 190 & \pm 140 & 2920 & $\pm 110^{\circ}$ \\
\hline Matière sèche totale $\left(\mathrm{g} . \mathrm{m}^{-2}\right)$ & 740 & \pm 150 & \pm 100 & 1160 & 1180 & \pm 110 \\
\hline
\end{tabular}


TABLEAU 3

Evolution de la matière sèche d'une graine (mg), des teneurs en protéines (\%) et en lipides (\%) au cours du remplissage (moyenne de 4 échantil lons \pm écart type).

Change in dry matter of one seed ( $\mathrm{mg})$, proteins (\%) and lipids (\%) during seed filling (mean of 4 samples \pm SD).

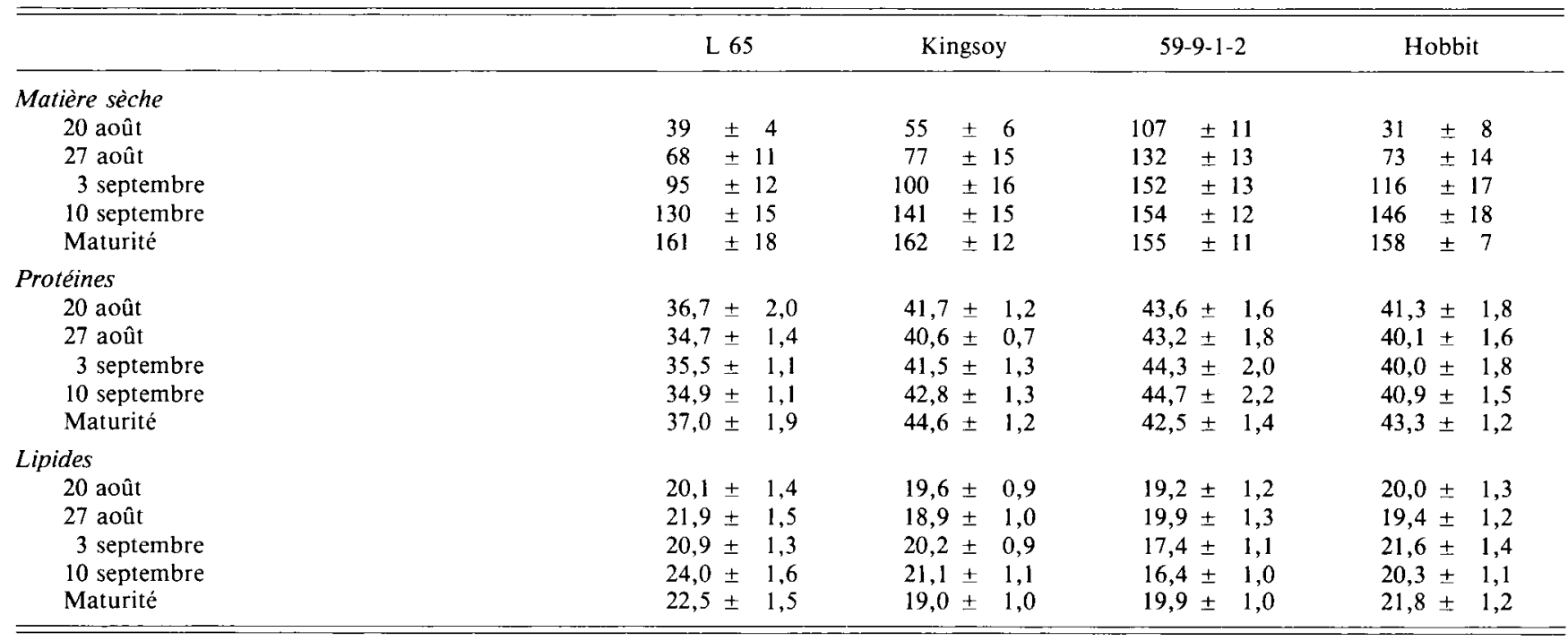

La matière sèche et la teneur en lipides et en protéines des graines ont été déterminées au cours d'une partie de leur développement (période du 20 août au 10 septembre) et à maturité (tabl. 3). Au début de la période étudiée, les graines des variétés " L 65 ", " Kingsoy " et " Hobbit " sont encore peu développées et, durant les semaines suivantes, leur masse s'accroît de façon linéaire. En revanche, les graines de la lignée " 59-9-1-2 ", plus hâtives, ont déjà atteint une taille conséquente.

Les taux de protéines et de lipides restent sensiblement constants durant toute la maturation, les moyennes respectives étant de 35,7 et $21,9 \mathrm{~g}$ pour $100 \mathrm{~g}$ de matière sèche pour « $\mathrm{L} 65$ ", 42,2 et 19,7 pour " Kingsoy », 43,6 et 18,5 pour « 59-9-1-2 », 41,2 et 20,6 pour « Hobbit ». Ces données permettent le calcul des équivalents-glucose nécessaires à la production de la matière sèche des graines, soit respectivement 0,$56 ; 1,05 ; 1,03 ; 1,01 \mathrm{~kg} \cdot \mathrm{m}^{-2}$ d'équivalentsglucose.

\section{B. Photosynthèse}

La photosynthèse nette des feuilles situées en haut du couvert a été mesurée de la floraison (début juillet) à la mi-septembre. Les variétés nodulées fixent sensiblement la même quantité de $\mathrm{CO}_{2}$, aussi, sur la figure 1, les valeurs moyennes de leur taux d'assimilation sont-elles comparées à celles de la lignée non nodulante " $\mathrm{L} 65$ ». Celle-ci assimile moins de $\mathrm{CO}_{2}$ (- 37 p. 100).

L'évaluation de la photosynthèse nette du couvert, réalisée selon la méthode WELLS et al. (1982), fait apparaître une capacité photosynthétique moindre pour la lignée « L 65 » (fig. 2), surtout au moment où apparaissent les premières graines ; son indice foliaire est nettement plus faible : 4,1 pour « L 65 » au lieu de 6 environ pour les variétés nodulées. Chez celles-ci, la photosynthèse nette, dont l'intensité dépasse parfois $20 \mu$ moles $\mathrm{CO}_{2} \cdot \mathrm{m}^{-2} \mathrm{~s}^{-1}$, ne présente pas de différences significatives. L'assimilation globale décroît relativement tôt, alors que les graines n'ont élaboré que le tiers ou le quart de leur masse définitive.

La quantité de carbone assimilée pendant la phase de remplissage, calculée à partir des courbes de la figure 2 et exprimée en $\mathrm{kg} \cdot \mathrm{m}^{-2}$ d'équivalentsglucose, est de 0,43 pour « L 65 », 0,70 pour « King-

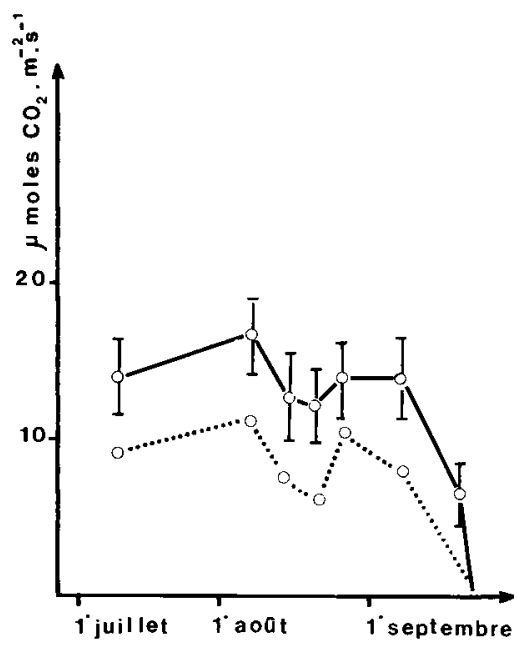

Figure 1

Evolution, à partir de la floraison, de la photosynthèse nette des feuilles du couvert végétal.

-. movenne des variétés nodulées, "Hobbit ", "Kingsoy ", " 59-9-1-2"

-..- lignée non nodulante « $L 65$ »

Net photosynthesis of canopy leaves from flowering to maturity - mean of nodulated varieties " Hobbit 》, "Kingsoy 》, « 59-9 $1-2 »$

--- non-nodulating line « $L 65 »$. 


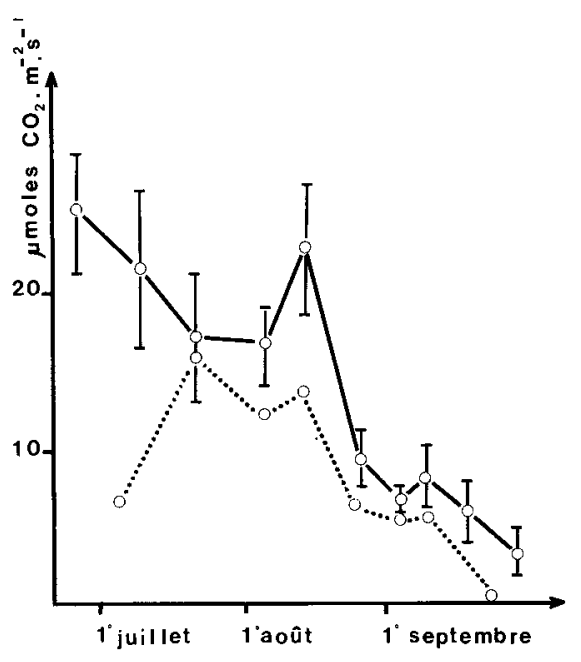

Figure 2

Evolution, à partir de la floraison, de la photosynthèse nette du couvert mesurée à l'aide de caissons transparents couvrant $0,5 \mathrm{~m}^{2}$ de sol. Même légende que pour la figure 1.

Net photosynthesis of canopy from flowering to maturity, measured with transparent chambers covering soil area of $0.05 \mathrm{~m}^{2}$. Legend as for figure 1 .

soy », 0,80 pour «59-9-1-2» et 0,83 pour « Hobbit ».

\section{Incorporation du ${ }^{14} \mathrm{CO}_{2}$ et marquage des consti- tuants des graines}

\section{Transferts des assimilats}

La détermination de la radioactivité présente dans les feuilles, 8 et $72 \mathrm{~h}$ après une charge de $30 \mathrm{mn}$ en ${ }^{14} \mathrm{CO}_{2}$, permet d'apprécier la capacité de la plante à transférer les assimilats, étant donné que les pertes par respiration sont faibles, environ 5 p. 100 (CALMES \& VIALA, 1985). Les chiffres regroupés dans le tableau 4 montrent qu'après $8 \mathrm{~h}$ de chasse la majeure partie du radiocarbone présent dans la feuille à la fin de l'incorporation a été transportée vers les autres parties de la plante. Les migrations semblent moins rapides dans la variété de type déterminé «Hobbit" (41 p. 100). Après $72 \mathrm{~h}$ tous les cultivars ont exporté au moins les deux tiers de la radioactivité.

\section{Marquage des divers constituants des graines}

Les graines analysées se situent à l'aisselle de la feuille ayant incorporé le ${ }^{14} \mathrm{CO}_{2}$. La répartition du radiocarbone entre les composés glucidiques, lipidiques et azotés est représentée sur la figure 3 pour les expérimentations effectuées le 20 août et sur la figure 4 pour celles du début du mois de septembre. Lors de la première incorporation, en fin de journée ( $8 \mathrm{~h}$ après la charge), la majeure partie du carbone 14 parvenu dans les graines est encore contenue dans les composés glucidiques solubles (60 p. 100) ; des autoradiogrammes ont montré que le saccharose était largement prédominant. Cependant les composés azotés en contiennent déjà 15 p. 100 répartis de façon à peu près égale entre les acides aminés libres et les protéines. Les différences entre les cultivars ne sont pas significatives ; seule la lignée "L 65 », non nodulante, présente un déficit de marquage des composés azotés (- 22 p. 100 par rapport à « Hobbit»). Les

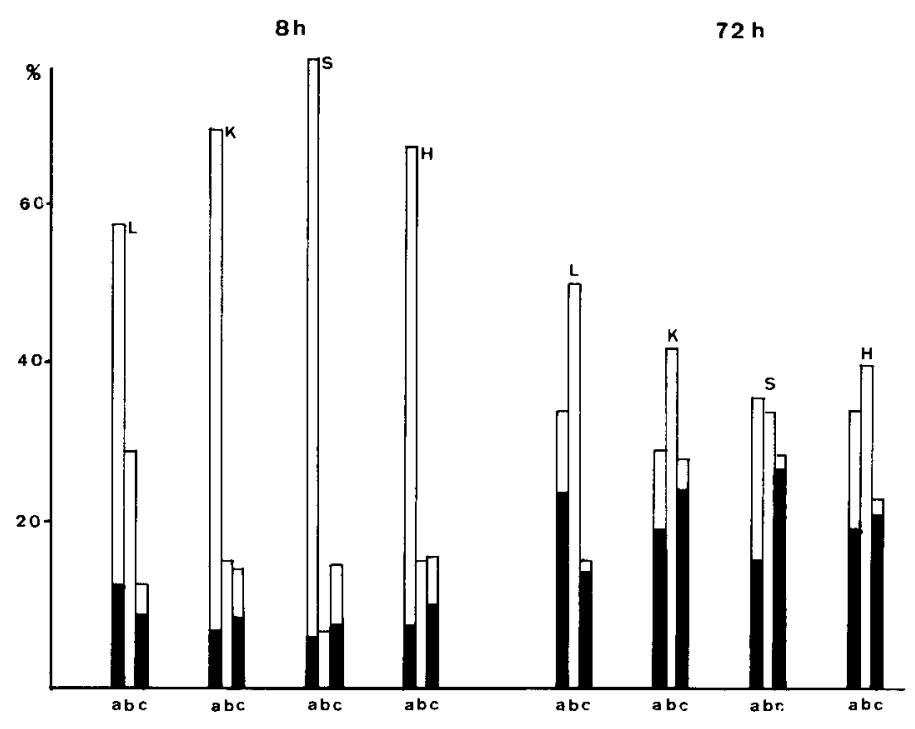

Figure 3

Répartition du radiocarbone des graines entre les divers groupes de composés, 8 et $72 \mathrm{~h}$ après une incorporation foliaire de ${ }^{14} \mathrm{CO}_{2}$ effectuée le 20 août. La radioactivité de l'ensemble de ces composés est prise égale à 100 .

$a$, composés glucidiques : $\square$ solubles, $\square$ insolubles; $b$, lipides ; c, composés azotés $: \square$ acides aminés libres, $\square$ protéines

$L$, « $L 65$ » non nodulante; $K$, «Kingsoy»; $S$, lignée semidéterminée "59-9-1-2》; $H$, « Hobbit 》.

Radiocarbon distribution in the seed between various groups of compounds, 8 and $72 \quad h$ after foliar ${ }^{14} \mathrm{CO}_{2}$ incorporation. August 20. Total radioactivity of these compounds $=100$. $a$, carbohydrates : $\square$ soluble; $\mathbf{\square}$ insoluble; $b$, lipids $c$, nitrogenous compounds; $\square$ free amino acids, $\mathbf{\square}$ proteins $L$, « $L 65$ » non-nodulating; $K$, « Kingsoy»; $S$, semi-determinate genotype « 59-9-1-2 "; $H$, « Hobbit ».

TABLEAU 4

Proportion de radiocarbone (en $\% d u{ }^{14} \mathrm{C}$ fixé en fin de charge) perdue par une feuille du couvert, au cours des 8 et 72 h qui suivent l'incorporation de ${ }^{14} \mathrm{CO}_{2}$. Celle-ci est effectuée le matin, vers $8 \mathrm{~h}$ (T.U.), à l'époque du grassissement des graines. Moyenne de 4 échantillons \pm écart type. Rate of radiocarbon loss (as \% of ${ }^{14} \mathrm{C}$ assimilated at the end of charge) by a canopy leaf, during the 8 or $72 \mathrm{~h}$ following ${ }^{14} \mathrm{CO}_{2}$ incorporation in the morning (about 8 h T.U.) during seed filling. Mean of 4 samples $\pm S D$.

\begin{tabular}{rccccc}
\hline \hline & L 65 & Kingsoy & 59-9-1-2 & Hobbit \\
\hline $8 \mathrm{~h}$ & $61,6 \pm 9,1$ & $53,4 \pm 7,0$ & $68,7 \pm 7,3$ & $41,4 \pm 12,3$ \\
$72 \mathrm{~h}$ & $65,4 \pm 9,4$ & $66,6 \pm 7,6$ & $77,0 \pm 10,6$ & $66,4 \pm 11,8$ \\
\hline
\end{tabular}


lipides renferment 16 p. 100 du radiocarbone dans les graines des variétés «Hobbit» et "Kingsoy», le double (30 p. 100) dans celles de la lignée «L 65 » et seulement 6 p. $100 \mathrm{chez}$ le semi-déterminé «59-9-1-2».

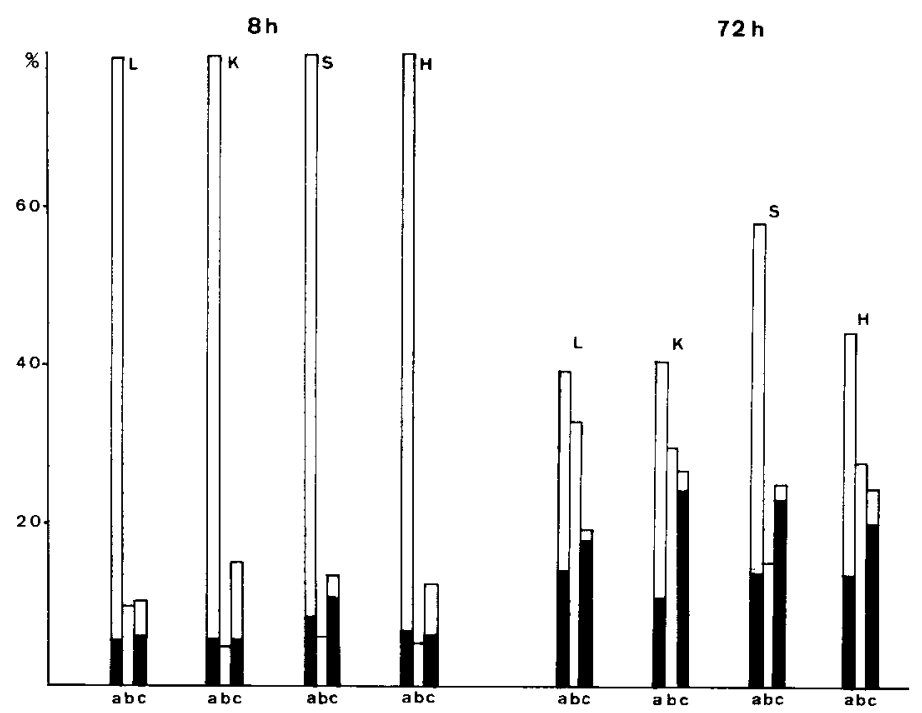

Figure 4

Répartition du radiocarbone des graines entre les divers groupes de composés, 8 et $72 \mathrm{~h}$ après une incorporation foliaire de ${ }^{14} \mathrm{CO}$ effectuée le 10 septembre (le 3 septembre pour la lignée « 59-9-1-2 》plus précoce). Même légende que pour la figure 3.

Radiocarbon distribution in the seed between various groups of compounds, 8 and $72 \mathrm{~h}$ after foliar ${ }^{14} \mathrm{CO}_{2}$ incorporation. September 10 (September 3 for earlier line « 59-9-1-2 »). Legend as for figure 3 .

Trois jours plus tard, les métabolites marqués parvenus dans les graines ont été utilisés pour les diverses synthèses et le carbone 14 est désormais incorporé dans des composés que l'on peut considérer comme stables. Les protéines en contiennent alors, en moyenne, 21 p. 100 et les acides aminés libres 4 p. 100. Ici encore, les variétés « Hobbit», « Kingsoy » et « 59-9-1-2 » ne présentent entre elles aucune différence significative ; en revanche, le déficit de la lignée «L 65 " non nodulante s'est accentué ( -30 p. 100 par rapport à « Hobbit »). Les lipides constituent les composés les plus marqués de la graine : la valeur maximale $(50$ p. 100 du radiocarbone) se situe toujours dans la lignée non nodulante, tandis que la valeur minimale (35 p. 100) concerne la lignée « 59-9-1-2 »; «Hobbit» et « Kingsoy » présentent des valeurs intermédiaires (42 p. 100). Des différences statistiquement significatives apparaissent entre les variétés.

Dans le cas de l'incorporation effectuée lorsque les graines ont pratiquement atteint leur masse définitive (fig. 4), le marquage des protéines est peu différent de celui observé précédemment ; en revanche, celui des lipides est beaucoup plus faible. La lignée non nodulante se distingue à nouveau par son aptitude à orienter le carbone vers les triglycérides.

\section{DISCUSSION}

Même si la photosynthèse nette des feuilles du sommet et celle de l'ensemble du couvert n'ont pas été mesurées de la même manière, la comparaison des graphiques des figures 1 et 2 montre que la majeure partie de l'assimilation est assurée par les feuilles de la "voûte » qui interceptent la plus grande partie de l'énergie lumineuse. Des indices foliaires très élevés ne paraissent donc pas constituer un avantage : ceci a déjà été montré à diverses reprises (BLANCHET \& Gelfi, 1979 ; PAUl et al., 1979). Cela explique aussi que la hauteur totale du couvert, très différente de "Kingsoy » à « Hobbit », n'influence guère la photosynthèse.

Il existe une bonne corrélation $(r=0,90)$ entre l'intensité de fixation du $\mathrm{CO}_{2}$ et la production des graines. Comme l'ont montré Wells et al. (1982), l'activité photosynthétique au cours de la phase de remplissage conditionne, pour une bonne part, le rendement. Cependant, la masse de carbone incorporée pendant la période de fructification, exprimée en équivalents-glucose, est inférieure à celle nécessaire à l'élaboration des graines (fig. 5). La redistribution d'assimilats, fixés antérieurement, est donc indispensable ; les réserves du feuillage (CALMÉS et al. 1983) et de l'ensemble de l'appareil végétatif sont alors mobilisées. Des observations encore provisoires laissent supposer, en fin de cycle, la participation substantielle d'assimilats stockés dans les racines.

Les teneurs en lipides et en protéines des graines sont pratiquement constantes pendant toute la durée de l'expérimentation : l'étude statistique des valeurs

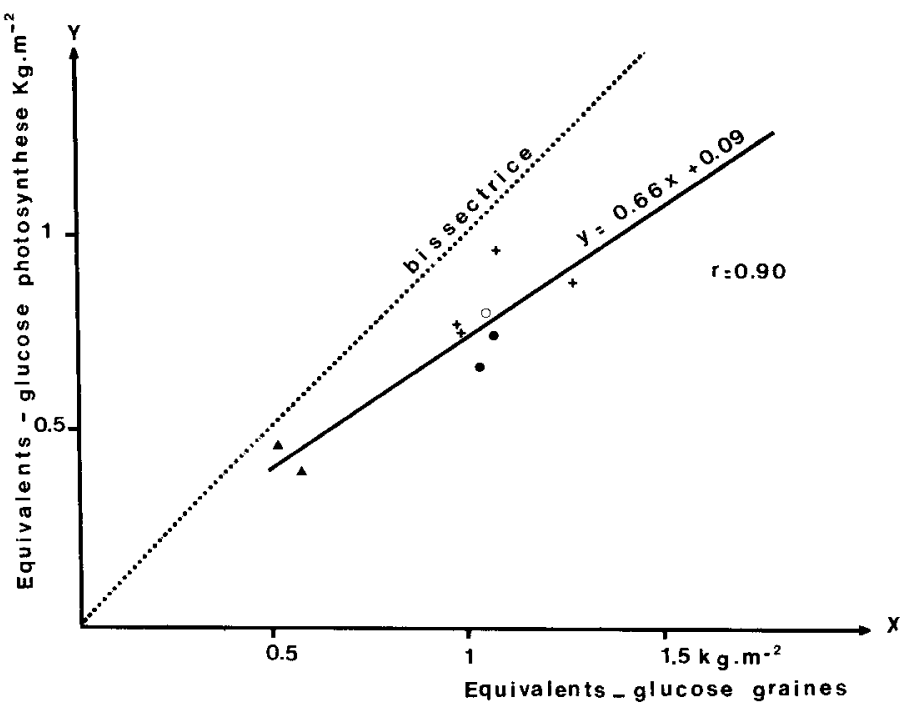

Figure 5

Graphique exprimant l'importance des équivalents-glucose $(x)$ des constituants des graines par rapport à ceux qui correspondent à l'ensemble du $\mathrm{CO}_{2}$ fixé pendant la période du remplissage (y).

$\Delta$, «L $65 » ; \bullet$, « Kingsoy »; O, " 59-9-1-2 »; +, « Hobbit ».

Diagram showing the importance of seed glucose-equivalents $(x)$ relative to glucose-equivalents of $\mathrm{CO}_{2}$ fixed during the seed-filling period $(y)$.

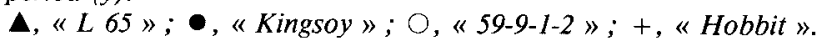


obtenues ne fait pas apparaître de différences significatives. Ainsi, les quantités accumulées augmentent proportionnellement à la masse de la graine dont la croissance pondérale est sensiblement linéaire (sauf pour la lignée « 59-9-1-2 », plus précoce) du 20 août au 10 septembre (tabl. 3). Par exemple, une graine de la variété «Hobbit » synthétise, le 20 août, environ $1,1 \mathrm{mg}$ de lipides et 2,3 de protéines et, le $10 \mathrm{sep}-$ tembre, 2,5 mg de lipides et 5,2 de protéines par jour.

La répartition de la radioactivité, lors des expériences de charge en ${ }^{14} \mathrm{CO}_{2}$, fait apparaître un marquage préférentiel des lipides de la graine où pourtant ils ne représentent que $20 \mathrm{p}$. 100 de la matière sèche ; la valeur moyenne du rapport de la radioactivité incorporée dans les lipides à celle des protéines varie de 1,75 le 20 août à 1,10 le 10 septembre. La distribution du carbone récemment assimilé évolue donc au cours de la formation des graines et n'est pas proportionnelle à l'accumulation de chaque catégorie de constituants. Il semble que la protéogenèse, dès le début du remplissage, bénéficie largement de la mobilisation de molécules carbonées et azotées préalablement stockées dans l'appareil végétatif. Par la suite, comme la masse des graines continue de croître alors que la photosynthèse nette s'atténue, l'hydrolyse des réserves accumulées ou celle des principaux constituants (Rubisco par exemple) devient prépondérante dans l'alimentation des gousses.

Les principales différences entre les cultivars concernent le taux de photosynthèse, les transferts vers les gousses et les teneurs en lipides et en protéines des graines. La lignée non nodulante « L 65 » se caractérise par une fixation de $\mathrm{CO}_{2}$ plus faible d'environ 30 p. 100 par rapport à la moyenne des trois autres. C'est l'une des conséquences de la déficience en azote (Grima-PetTEnATI, 1985) et peut-être aussi du nombre réduit des graines, la photosynthèse étant régulée par la demande en assimilats (PINTO, 1980).

Les transferts vers les graines sont, au 20 août, plus faibles chez "Hobbit " et plus importants pour les lignées « L 65 » non nodulante et surtout « 59-9-1-2 »; mais ceci est dû probablement à ce que l'âge physiologique n'est pas le même. "Hobbit " (groupe de précocité III) est à un stade plus jeune que la lignée « L 65 » et surtout « 59-9-1-2 » (groupe de précocité I).
Dans la composition finale des graines, la principale différence significative concerne « $L 65$ », plus riche en huile et plus pauvre en protéines que les trois autres. L'alimentation azotée joue donc un rôle plus déterminant que le type de croissance.

En conclusion et du point de vue agronomique, la morphologie de ces trois types de croissance déterminée, semi-déterminée ou indéterminée ne semble pas influencer beaucoup le fonctionnement des couverts et l'utilisation des assimilats, du moins dans les bonnes conditions culturales réalisées ici (BEAVER et al., 1985 ; BLANCHET et al., 1986). Les redistributions d'assimilats sont sans doute un peu plus importantes chez le type indéterminé, ce qui peut le rendre moins tributaire des conditions d'environnement pendant la phase de remplissage des graines. La tendance à l'exubérance de l'appareil végétatif, qui lui confère une forte sensibilité à la verse, est un caractère agronomique défavorable, notamment en culture irriguée. On peut supposer qu'il est plutôt adapté à des environnements contraignants, tandis que le type déterminé conviendrait mieux à des milieux favorables, en particulier à l'irrigation; le type semi-déterminé serait intermédiaire. Le début plus précoce du remplissage des graines chez ces deux derniers types semble avantageux : l'énergie lumineuse est, en effet, de moins en moins utilisée au fur et à mesure que le cycle de développement se prolonge (diminution de la température et, probablement, ralentissement des translocations : PlANCHON, 1986). Ainsi, «Hobbit » n'a pas très bien utilisé l'ensoleillement reçu en fin de cycle, tandis que la lignée « 59-9-1-2 » paraît un modèle intéressant, capable de réaliser en un cycle court des biosynthèses efficaces.

Reçu le 5 juin 1986. Accepté le 3 octobre 1986.

\section{REMERCIEMENTS}

Nous remercions MM. BERNARd (U.S.D.A., Urbana) et VIDAL (I.N.R.A., Montpellier) de nous avoir fourni gracieusement les graines des lignées « $L$ 65 》 et « 59-9-1-2 》 et le CETIOM de son appui technique et financier dans cette recherche. M. Pons, de l'Institut de Mécanique des Fluides de Toulouse, a bien voulu nous aider à mettre au point l'appareillage de mesure de photosynthèse.

\section{RÉFÉRENCES BIBLIOGRAPHIQUES}

Beaver J. S., Cooper R. L., Martin R. J., 1985. Dry matter accumulation and seed yield of determinate and indeterminate soybeans. Agron. J., 77, 675-679.

Blanchet R., Gelfi N., 1979. Influence de réductions de la surface foliaire sur la croissance, le développement et la production d'un soja de type indéterminé (Glycine max (L.) Merril, cv. Amsoy 71). C. R. Acad. Sci., Paris, série D, 289, 299-302.

Blanchet R., Merrien A., Gelfi N., Cavalie G., Courtiade B., Puech J., 1982. Estimation et évolution comparée de l'assimilation nette de couverts de maïs (Zea Mays L.), tournesol (Helianthus annuus L.) et soja (Glycine max (L.) Merril), au cours de leurs cycles de développement. Agronomie, 2, 149-154.

Blanchet R., Bouniols A.. Constant S., Gelfi N., Marty J. R., Puech J., 1986. Biologie et formation du rendement de sojas déterminés et indéterminés, en bonnes conditions culturales. Eurosoya, 4, 10-17.
Bouniols A., Puech J., Chalamet A., Mondies M., 1985. Influence des conditions d'alimentation hydrique ou (et) azotée, à différents stades du développement, sur la production de grains et la nutrition azotée du soja. Eurosova, 3, 55-61.

Calmés J., Naudy M., Viala G., 1983. Protéogenèse des graines de soja Glycine max (L.), Merr. : origine des acides aminés. Agronomie, 3, 273-278.

Calmés J., Viala G., 1985. Estimation de la respiration non-photorespiratoire pour une feuille de soja exposée à la lumière. $C . R$. Acad. Sci., Paris, série III, 301, 95-98.

Calmés J., Viala G., Gelfi N., Blanchet R., 1985. Influence d'un déficit hydrique sur trois variétés de soja : effet sur la protéogenèse des graines. Agronomie, 5, 169-176.

Cooper R. L., 1981. Development of short-statured soybean cultivars. Crop Sci., 2, 127-131. 
Egli D. B., Guffy R. D., Meckel L. W., Leggett J. E., 1985. The effect of source-sink alterations on soybean seed growth. Ann. Bot., 55, 395-402.

Fishwick M. J., Wright A. J., 1977. Comparison of methods for the extraction of plant lipids. Phytochemistry, 16, 1507-1510.

Grima-Pettenati J., 1985. Effets d'une privation d'azote sur la nutrition carbonée et la dégradation des protéines foliaires chez le soja. Thèse Doct. $3^{\mathrm{e}}$ cycle, Université Paul Sabatier, Toulouse, $116 \mathrm{p}$.

Layzell D. B., La Rue T. A., 1982. Modeling C and N transport to developing soybean fruits. Plant Physiol., 70, 1290-1298.

Mazliak P., 1968. Le métabolisme des lipides dans les plantes supérieures. Monographies de Physiologie Végétale. Masson, Paris, $223 \mathrm{p}$

Murphy D. J., Leech R. M., 1981. Photosynthesis of lipids from ${ }^{14} \mathrm{CO}_{2}$ in Spinacia oleracea. Plant Physiol., 68, 762-765.

Naudy-de Serres M., 1985. Composés azotés foliaires du soja : leur rôle dans la nutrition des gousses. Thèse Doct. $3^{\mathrm{e}}$ cycle, Université Paul Sabatier, Toulouse, 64 p.

Ohlrogge J. B., Kuo T. M., 1984. Control of lipid synthesis during soybean seed development : enzymic and immunochemical assay of acyl carrier protein. Plant Physiol., 74, 622-625.

Pate J. S., 1980. Transport and partitioning of nitrogenous solutes. Ann. Rev. Plant Physiol., 31, 314-340.

Paul M. H., Planchon C., Ecochard R., 1979. Etude des relations entre le développement foliaire, le cycle de développement et la productivité chez le soja. Ann. Amélior. Plantes, 29, 478-492.
Penning De Vries F. W. T., Brunstig A. H. M., Van Laar H. H., 1974. Products, requirements and efficiency of biosynthesis : a quantitative approach. $J$. Theor. Biol., 45, 339-377.

Pinto M., 1980. Régulation de la photosynthèse par la demande d'assimilats : mécanismes possibles. Photosynthetica, 14, 611-637.

Planchon C., 1986. Ecophysiologie du soja : adaptation aux conditions d'éclairement et de température, in Le Soja: physiologie de la plante et adaptation aux conditions francaises, CETIOM et I.N.R.A. éd., Informations techniques, 73-78.

Privett O. S., Dougherty K. A., Erdahl W. L., Stolyhwo A., 1973. Studies on the lipid composition of developing soybeans. J. Am. Oil Chem. Soc., 50, 516-520.

Rubel A., Rinne R. W., Canvin D. T., 1972. Protein, oil and fatty acid in developing soybean seeds. Crop Sci., 12, 739-741.

Salado-Navarro L. R., Hinson K., Sinclair T. R., 1985. Nitrogen partitioning and dry matter allocation in soybeans with different seed protein concentration. Crop Sci., 25, 451-455.

Serres E., Calmés J., Viala G., Cavalié G., 1985. Uréides et asparagine chez deux variétés de soja cultivées au champ : stockage dans la tige et utilisation. Agronomie, 5, 899-904.

Shimshi D., 1969. A rapid field method of measuring photosynthesis with labelled carbon dioxyde. Is. J. Bot., 20, 381-401.

Vidal A., Hanafiah T. O., 1985. Influence du type de croissance sur la morphologie et la floraison du soja (Glycine max (L.) Merril). Eurosoya, 3, 62-70.

Wells R., Schulze L. L., Ashley D. A., Boerma H. R., Brown R. H., 1982. Cultivar differences in canopy apparent photosynthesis and the relationship to seed yield in soybeans. Crop Sci., 22, 886-890. 